\title{
Viscoelasticity of a Nonionic Lamellar Phase
}

\author{
B. Medronho, ${ }^{*, \dagger}, \pitchfork$ Maria G. Miguel, ${ }^{\ddagger}$ and U. Olsson ${ }^{\dagger}$ \\ Physical Chemistry 1, Center of Chemistry and Chemical Engineering, Lund University, Box 124, \\ 22100 Lund, and Department of Chemistry, University of Coimbra, 3004-535, Coimbra, Portugal
}

Received December 13, 2006. In Final Form: March 15, 2007

\begin{abstract}
The linear viscoelastic properties of a nonionic lamellar phase in C-orientation were studied as a function of temperature by small-amplitude oscillatory measurements in the frequency range $0.5-5 \mathrm{~Hz}$. An almost solidlike elastic response was observed at all studied temperatures, from 42 to $20{ }^{\circ} \mathrm{C}$. In this range, the elastic modulus was found to increase strongly with decreasing temperature. The elasticity is attributed to screw dislocations connecting layers in the stack, and the data thus suggest that the density of screw dislocations decreases with increasing temperature. The lamellar phase forms an "onion" texture when continuously sheared at lower temperatures. It is argued that a possible origin for the shear-induced "onion" texture is the instability of the screw dislocations in shear flow. By ${ }^{2} \mathrm{H}$ NMR experimentation, we also find the formation of a random mesh phase at lower temperatures. The presence of equilibrium bilayer perforations, however, does not correlate with the "onion" stability.
\end{abstract}

\section{Introduction}

The shear-induced transformations and ordering in "complex fluids", such as in lyotropic liquid-crystalline phases, thermotropic liquid crystals, and polymers melts is of both practical and fundamental interest. ${ }^{1-5}$ One of the most interesting shear effects is the transformation from planar lamellar phases to multilamellar vesicles (MLV or "onions"). Although there is a lot of work concerning the shear-induced formation of $\mathrm{MLV},{ }^{6-12}$ the mechanism governing this transformation is not yet fully understood. The different stationary states induced by shear, like MLVs, are separated by out-of-equilibrium transitions which result from hydrodynamical instabilities within the lamellar phase. This seems to be a complex process and depends, for instance, on the applied shear stress and shear strain. ${ }^{13,14}$ The transition from planar lamellae to a MLV phase has been proposed to be a consequence of a mechanical flow instability of the planar lamellae at higher shear rates. ${ }^{15-18}$ For instance, Zilman and Granek ${ }^{17}$ proposed that the coupling of short-wavelength thermal undulations and strain lead to a coherent stripe buckling of the lamellae that eventually results in MLV formation.

* Bruno.Medronho@fkem1.lu.se.

†und University.

$\doteqdot$ University of Coimbra. 223.

(2) Colby, R.; Ober, C.; Gilmor, J.; Connelly, R.; Duong, T.; Galli, G.; Laus, M. Rheol. Acta 1997, 36, 498.

(3) Larson, R.; Winey, K.; Patel, S.; Watanabe, H.; Bruinsma, R. Rheol. Acta 1993, 32, 245 .

(4) Paasch, S.; Schambil, F.; Schwuger, J. Langmuir 1989, 5, 1344.

(5) Németh, Z.; Halász, L.; Pálinkás, J.; Bóta, A.; Horányi, T. Colloids Surf., A 1998, 145, 107.

(6) Richtering, W. Curr. Opin. Colloid Interface Sci 2001, 6, 446.

(7) Mortensen, K. Curr. Opin. Colloid Interface Sci 2001, 6, 140.

(8) Butler, P. Curr. Opin. Colloid Interface Sci. 1999, 4, 214.

(9) Berni, M. G.; Lawrence, C. J.; Machin, D. Curr. Opin. Colloid Interface Sci. 2002, 98, 217.

(10) Diat, O.; Roux, D. J. Phys. II 1993, 3, 9.

(11) Diat, O.; Roux, D.; Nallet, F. J. Phys. II 1993, 2, 1427.

(12) Medronho, B.; Fujii, S.; Richtering, W.; Miguel, M. G.; Olsson, U. Colloid Polym. Sci. 2005, 284, 317.

(13) Bergenholtz, J.; Wagner, N. J. Langmuir 1996, 12, 3122.

(14) Schmidt, G.; Muller, S.; Schmidt, C.; Richtering, W. Rheol. Acta 1999, 38,486 .

(15) Aurnhammer, G.; Brand, H.; Pleiner, H. Phys. Rev. E 2002, 66, 0061707.

(16) Marlow, S.; Olmsted, P. Eur. Phys. J. E 2002, 8, 485.

(17) Zilman, A.; Granek, R. Eur. Phys. J. B 1999, 11, 593.

(18) Soddemann, T.; Auerhammer, G.; Guo, H.; Dünweg, B.; Kremer, K. Eur. Phys. J. E 2004, 13, 141.
Other authors proposed that shear-induced transformations are also dependent on defects, ${ }^{19-23}$ which are expected to exist in these systems in order to relax external and internal stresses. These low-energy defects can assume different topologies within a smectic lamellar system; for instance, the lamellar system which is a one-dimensional (1D) crystal of parallel layers that are twodimensional (2D) liquids possesses a translational symmetry and thus exhibits dislocations (screw and edge dislocations). , $21,23,24^{2}$ The influence of flow in these defective systems is poorly understood, but indeed defects strongly influence the rheological properties. In theory, a 1D stack of $2 \mathrm{D}$ fluid layers ( $\mathrm{L}_{\alpha}$ phase) in a perfectly ordered and oriented state should not be able to store elastic energy, which means that a liquid response would be expected since the layers should be free to slide past one another in the shear flow axis. Oswald and Allain ${ }^{19}$ have shown that the line tension of a screw, tending to maintain the helical axis perpendicular to the layers, is opposed by viscous forces. They predict a critical shear rate beyond which the defect becomes unstable and should disappear, returning the lamellar phase to a " $\mathrm{C}$ " orientation (the normal to the layers along the velocity gradient) without defects. Basappa et al. ${ }^{25}$ showed that a defective lamellar system can be stabilized by particles ${ }^{26}$ giving rive even to higher elastic properties, higher storage modulus $\left(G^{\prime}\right)$, when compared to the same system without added particles. They also argue about the pre-shear treatment in their system, finding a decrease in $G^{\prime}$ when the system was pre-sheared.

In these two examples, it is clear that the presence and stability of defects change dramatically the rheological properties of a lamellar system. In the present paper, we report on the temperature dependence of the linear viscoelastic properties of a model nonionic lamellar phase, composed of the nonionic surfactant $\mathrm{C}_{10} \mathrm{E}_{3}$ in water. The motivation behind the work is to understand the properties of lamellar phases in general, but in particular, we

(19) Oswald, P.; Allain, M. J. Colloid Interface Sci. 1988, 126, 45.

(20) Penfold, J.; Staples, E.; Lodhi, A.; Tucker, I.; Tiddy, G. J. Phys. Chem. B 1997, 101, 66

(21) Blanc, C.; Meyer, C.; Asnacios, S.; Kleman, M.; Lelidis, I.; Marin, J. Philos. Mag. Lett. 2005, 85, 641.

(22) Moreau, P.; Navailles, L.; Giermanska-kahn, J.; Mondain-Monval, O ; Nallet, F.; Roux, D. Europhys. Lett. 2006, 73, 49.

(23) Dhez, O.; Nallet, F.; Diat, O. Europhys. Lett. 2001, 55, 821.

(24) Kleman, M. Rep. Prog. Phys. 1989, 52, 555.

(25) Basappa, G.; Kumaran, V.; Nott, P.; Ramaswamy, S.; Naik, V.; Rout, D. Eur. Phys. J. B 1999, 12, 269.

(26) Zapotocky, M.; Ramos, L.; Poulin, P.; Lubensky, T.; Weitz, D. Science 1999, 283, 209. 
want to investigate possible correlations among the details of the lamellar phase structure, including equilibrium "defects", and the formation of shear-induced multilamellar vesicles, often referred to as "onions". In the water $-\mathrm{C}_{10} \mathrm{E}_{3}$ system, "onions" can form at lower temperatures while the lamellar structure remains stable in shear flow at higher temperatures near the lamellar-to-"sponge" phase transition. ${ }^{27,28}$ Onion formation in this system has been studied both in continuous shear and with large-amplitude oscillations. ${ }^{29,30}$

\section{Experimental Section}

Materials. Triethylene glycol mono $n$-decyl ether $\left(\mathrm{C}_{10} \mathrm{E}_{3}\right)$ was purchased from Nikko Chemical Co. (Tokyo, Japan) with a purity higher than $99.8 \%$. Deuterium oxide $\left(\mathrm{D}_{2} \mathrm{O}\right)$ was obtained from Sigma Chemicals (Steinheim, Germany). All samples were prepared by weighing the desired amount of surfactant and water, followed by a mixing procedure in the vortex, centrifuge, and oscillatory platform. All samples were prepared with $\mathrm{D}_{2} \mathrm{O}$.

${ }^{2}$ H NMR. NMR experiments were carried out in a Bruker DMX100 NMR spectrometer operating at $15.371 \mathrm{MHz}$ in a $2.3 \mathrm{~T}$ magnetic field. The quadrupolar splitting values in the lamellar and randommesh phases were recorded using the quadrupolar-echo sequence. The temperature of the sample was achieved and maintained using an airflow system, to an accuracy of $\pm 1{ }^{\circ} \mathrm{C}$.

Rheometry. The rheology experiments were performed on a Physica UDS 200 rheometer using the cone and plate geometry (MK22/M, $1^{\circ}$ cone angle). The instrument is equipped with a temperature control unit that was calibrated to give a temperature in the sample chamber within $0.1^{\circ} \mathrm{C}$ of the set value. A solvent trap was used to prevent water evaporation. A pre-shear treatment was applied at $42^{\circ} \mathrm{C}$ with a constant shear rate of $10 \mathrm{~s}^{-1}$ in order to have a well-defined and reproducible initial state in the experiments. The viscoelastic properties of the system were studied by small-frequency oscillatory tests where the linear regime was previously verified by stress sweep tests at the different temperatures used. All oscillatory tests were performed under controlled stress conditions.

\section{Results and Discussion}

Viscoelasticity and Screw Dislocations. The phase diagram of the $\mathrm{C}_{10} \mathrm{E}_{3}$-water system can be found elsewhere, ${ }^{31}$ and the concentration used in the present work is within the described lamellar phase. Here, we mainly focus on a lamellar phase composition of $40 \mathrm{wt} \%$ of $\mathrm{C}_{10} \mathrm{E}_{3}$ in $\mathrm{D}_{2} \mathrm{O}$, corresponding to a surfactant volume fraction of approximately 0.44 . Because of the structural anisotropy, the rheology of a lamellar phase depends on the orientation in the rheometer, and it is therefore preferred to work with a well-oriented lamellar phase. For a $40 \%$ sample, a lamellar phase oriented with normal in the gradient direction (C-orientation) is obtained when shearing the sample around 42 ${ }^{\circ} \mathrm{C}$. In Figure 1 , we show the evolution of the viscosity at $42{ }^{\circ} \mathrm{C}$ of an initially polycrystalline sample when sheared at a constant rate of $10 \mathrm{~s}^{-1}$. As the lamellar domains orient in the shear flow, the apparent viscosity decreases. After approximately 3000 strain units $(300 \mathrm{~s})$, the viscosity has reached a low value, indicating that essentially a single orientation has been obtained, which in previous scattering experiments has been identified with the C-orientation. ${ }^{32}$ We note that viscosity increases slowly after ca.

(27) Le, T. D.; Olsson, U.; Mortensen, K.; Zipfel, J.; Richtering, W. Langmuir 2001, 17, 999 .

(28) Oliviero, C.; Coppola, L.; Gianferri, R.; Nicotera, I.; Olsson, U. Colloids Surf., A 2003, 228, 85 .

(29) Nettesheim, F.; Zipfel, J.; Olsson, U.; Renth, F.; Lindner, P.; Richtering, W. Langmuir 2003, 19, 3603.

(30) Nettesheim, F.; Olsson, U.; Lindner, P.; Richtering, W. J. Phys. Chem. B 2004, 20, 6328.

(31) Ali, A.; Mulley, B. J. Pharm. Pharmacol. 1978, 30, 205.

(32) Zipfel, J.; Nettesheim, F.; Lindner, P.; Le, T. D.; Olsson, U.; Richtering, W. Europhys. Lett. 2001, 53, 335.

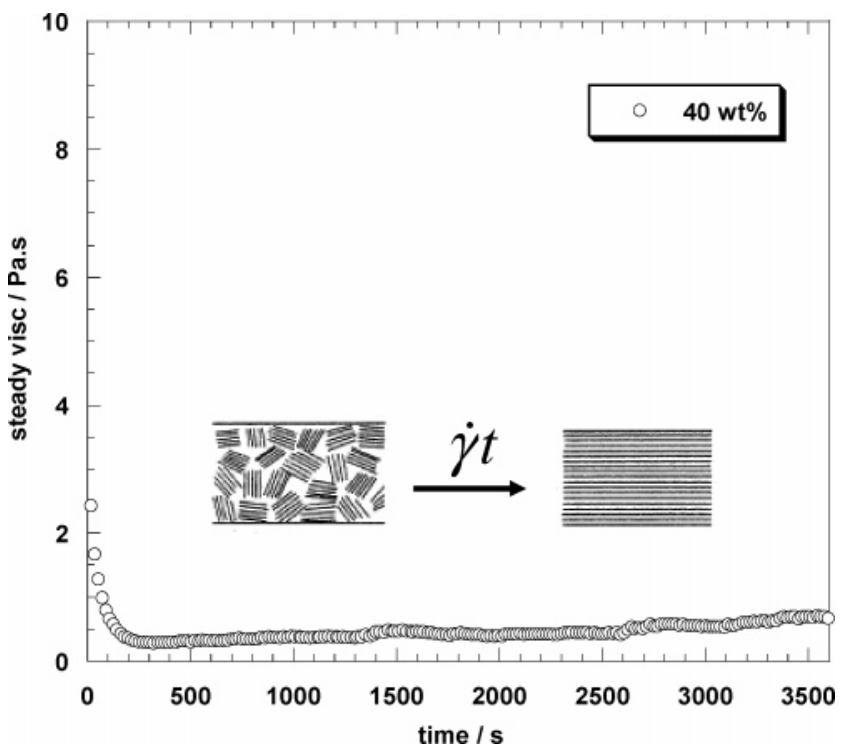

Figure 1. Steady viscosity as a function of time for the $40 \mathrm{wt} \%$ $\mathrm{C}_{10} \mathrm{E}_{3}$ sample as loaded in a polycrystalline state. This pre-shear treatment (shear rate of $10 \mathrm{~s}^{-1}$ during $1 \mathrm{~h}$ at $42{ }^{\circ} \mathrm{C}$ ) was applied to all samples before the oscillatory tests, to ensure, as much as possible, reproducible initial conditions in all cases.

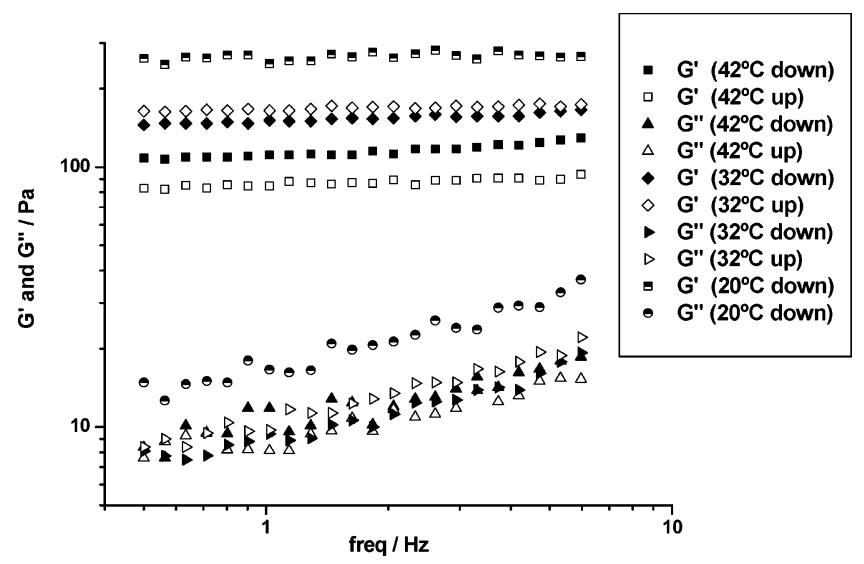

Figure 2. Linear moduli, $G^{\prime}$ and $G^{\prime \prime}$, vs frequency for the $40 \mathrm{wt}$ $\% \mathrm{C}_{10} \mathrm{E}_{3}$ sample at three selected temperatures: 20,32 , and $42{ }^{\circ} \mathrm{C}$. Data at 42 and $32{ }^{\circ} \mathrm{C}$ were repeated twice as the temperature was first lowered from $42{ }^{\circ} \mathrm{C}$ and then after $20^{\circ} \mathrm{C}$ increased again back to $42{ }^{\circ} \mathrm{C}$

$300 \mathrm{~s}$, which in part may be due to evaporation. This was not investigated further, as the main importance here is that the viscosity is low, demonstrating the presence of a $\mathrm{C}$-orientation. This pre-shear protocol was used for all samples, as it allows a well-defined and reproducible initial state.

The linear viscoelasticity was investigated by small-amplitude oscillations at different temperatures in the range $20-42^{\circ} \mathrm{C}$, for the frequency range $0.5-5 \mathrm{~Hz}$. In Figure 2, we present the frequency dependence of the storage $\left(G^{\prime}\right)$ and loss $\left(G^{\prime \prime}\right)$ moduli, respectively, at three temperatures: 20,32 , and $42{ }^{\circ} \mathrm{C}$. Data at 42 and $32{ }^{\circ} \mathrm{C}$ were repeated twice, as repeated experiments were performed with a temperature cycle. The temperature was first lowered from $42{ }^{\circ} \mathrm{C}$ and then after $20^{\circ} \mathrm{C}$ increased again back to $42{ }^{\circ} \mathrm{C}$. The behavior is essentially reversible upon temperature cycling. A slight drift may be observed, and $G^{\prime}$ is ca. $20 \%$ lower when returning to $42{ }^{\circ} \mathrm{C}$ compared to the first initial measurement. This may, however, be due to a slight loss of sample, as the second experiment was performed $3 \mathrm{~h}$ after the first one (the temperature cycle involved measurements at several other temperatures). $G^{\prime}$ is approximately an order of magnitude larger 
than $G^{\prime \prime}$. Within the frequency window, $G^{\prime}$ is essentially frequency-independent, while $G^{\prime \prime}$ approximately scales with $\omega^{0.4}$. Both $G^{\prime}$ and $G^{\prime \prime}$ decrease strongly with increasing temperature. At 20 and $42{ }^{\circ} \mathrm{C}$, the values differ approximately by a factor of 3. The spectra at different temperatures differ only by a proportionality constant, while the frequency dependence remains essentially the same. For our frequency window, we can write $G^{\prime} \approx G_{0}$ and $G^{\prime \prime} \sim G_{0} \omega^{0.4}$, where the main temperature dependence lies in the modulus $G_{0}$.

The fact that we observe here a dominating elastic response may at first seem surprising. For a lamellar phase in the C-orientation, we expect bilayers to be able to slide freely past each other, rheologically behaving as a Newtonian liquid. Howerer, the results indicate the presence of bilayers that are somehow connected.

Neighboring bilayers may be connected by passages as in the "sponge" phase. However, such passages cannot explain the observed elasticity. First of all, the passages are expected to occur more frequently close to the lamellar-to-"sponge" transition at higher temperatures. Second, since the bilayers are fluid, passages can freely slide, which in fact is the reason why, in spite of multiply connected bilayer structure, a sponge phase behaves as a Newtonian liquid. ${ }^{33}$ Lamellar phases contain thermally excited edge and screw dislocations that are expected to influence the flow. Meyer et al. ${ }^{34}$ have proposed that it is the concentration and mobility of screw dislocations that governs the viscosity of the lamellar phase in the C-orientation. Screw dislocations are oriented in the direction parallel to the director (average bilayer normal) of the phase and connects a stack of layers. Finite-sized screws occur in pairs, connected by edge dislocations, forming screw dislocation loops. At rest, the screw axis is parallel with the average layer normal. A shear deformation results in a tilt of the screw axis, which presumably is the origin of the storage of elastic energy. The elastic modulus is expected to depend on the length and the number density of screws, the product of which can be defined as the screw density, $\rho_{\mathrm{s}}$. If the elastic energy stored in a shear deformation is proportional to the total length of screw dislocations, we expect $G_{0} \sim \rho_{\mathrm{s}}$. The observed strong temperature dependence of $G_{0}$ thus indicates that $\rho_{\mathrm{s}}$ increases with decreasing temperature. This is consistent with the recent findings of Navalliers et al. ${ }^{22}$ In a similar system, containing the nonionic surfactant $\mathrm{C}_{12} \mathrm{E}_{5}$ mixed with a phospholipid, they observe an increased screw density as the temperature was lowered in the lamellar phase.

The variation of the viscoelastic spectrum with temperature is reversible. This was already demonstrated in Figure 2. To show this reversibility for a wider range of temperatures, we present in Figure 3 the temperature dependence of $G^{\prime}$ and $G^{\prime \prime}$, for a fixed oscillation frequency of $4 \mathrm{~Hz}$. The data in Figure 3 represent the results of two separate experimental runs. Square and circular symbols represent an experimental run starting at $42{ }^{\circ} \mathrm{C}$ with the pre-shear, followed by oscillatory experiments. The temperature was then lowered in steps to $20^{\circ} \mathrm{C}$, after which the temperature was stepped up again. The triangular symbols represent a second run on a different sample, involving only a stepwise decrease of the temperature, which was now extended down to $2{ }^{\circ} \mathrm{C}$. In Figure 3, we see that not only do we observe reversibility as we vary the temperature, but also the data can be reproduced with a new sample. In both experimental runs, the viscoelastic spectrum in the range $0.5-5 \mathrm{~Hz}$ was recorded at each temperature (not shown). The spectra remained qualitatively the same over the whole large temperature range.

(33) Snabre, P.; Porte, G. Europhys. Lett. 1990, 13, 641-645.

(34) Meyer, C.; Asnacios, S.; Kleman, M. Eur. Phys. J. E 2001, 6, 245.

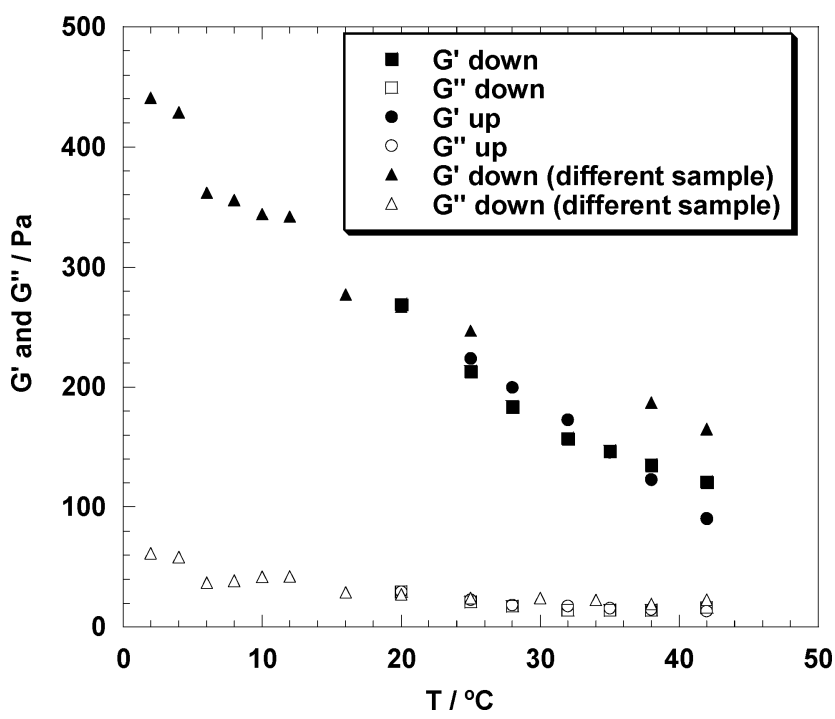

Figure 3. Linear moduli $G^{\prime}$ (filled symbols) and $G^{\prime \prime}$ (open symbols), recorded at $4 \mathrm{~Hz}$, vs temperature. The figure contains results from two samples. With one sample (triangles), the temperature was stepwise lowered from 42 to $2^{\circ} \mathrm{C}$. In the second sample, a temperature cycle was performed. Starting from $42^{\circ} \mathrm{C}$, the temperature was first stepwise lowered to $20^{\circ} \mathrm{C}$ (squares), then increased back to $42{ }^{\circ} \mathrm{C}$ (circles), and the moduli were measured at the same temperatures as during the temperature decrease.

The Random-Mesh Phase. Another structural property of lamellar phases, which for non-ionic surfactants is known to depend on temperature, is bilayer perforations. In order to see whether the presence of perforations may influence the relative stability of onions and planar lamellae under shear, we have performed ${ }^{2} \mathrm{H}$ NMR experiments using $\mathrm{D}_{2} \mathrm{O}$ at different temperatures. The NMR experiment allows us to detect perforations, and the question is whether there exists a correlation between the presence of perforations and the onion stability. Recent detailed structural studies have resulted in a number of reports on bilayer perforations and lamellar-mesh phases, starting with the pioneering work of Holmes et al. ${ }^{35-38}$ In several nonionic surfactant water lamellar phases, as in the water $-\mathrm{C}_{12} \mathrm{E}_{5}$ and water $-\mathrm{C}_{16} \mathrm{E}_{6}$ systems, the bilayers appear to be highly perforated at lower temperatures while being essentially without perforations at higher temperatures. Signatures of perforations are a broad diffuse scattering maximum at $q$-vectors around the first-order quasi Bragg peak, ${ }^{39-41}$ and a low value of the structural order parameter measured, e.g., by ${ }^{2} \mathrm{H}$ NMR. ${ }^{42}$

Interestingly, a random mesh structure also occurs in the present water $-\mathrm{C}_{10} \mathrm{E}_{3}$ lamellar phase; however, perforations appear first below ca. $10^{\circ} \mathrm{C}$. In Figure 4, we present how the ${ }^{2} \mathrm{H}$ quadrupolar splitting, $\Delta v_{\mathrm{Q}}$, from the deuterated water varies with temperature for the $40 \mathrm{wt} \%$ sample. Cooling from $42{ }^{\circ} \mathrm{C}, \Delta v_{\mathrm{Q}}$ first increases, as is normally found in surfactant liquid crystalline phases, due to an increased local ordering of water molecules hydrating the

(35) Funari, S. S.; Holmes, M. C.; Tiddy, G. J. T. J. Phys. Chem. 1994, 98, 3015 .

(36) Funari, S. S.; Holmes, M. C.; Tiddy, G. J. T. J. Phys. Chem. 1992, 96, 11029.

(37) Fairhurst, C.; Holmes, M.; Leaver, M. Langmuir 1997, 13, 4964.

(38) Burgoyne, J.; Holmes, M. C.; Tiddy, G. J. T. J. Phys. Chem. 1995, 99, 6054.

(39) Imai, M.; Saeki, A.; Teramoto, T.; Kawaguchi, A.; Nakaya, K.; Kato, T.; Ito, K. J. Chem. Phys. 2001, 115, 10525.

(40) Imai, M.; Sakai, K.; Kikuchi, M.; Nakaya, K.; Saeki, A.; Teramoto, T. J. Chem. Phys. 2005, 122, 214906.

(41) Imai, M.; Kawaguchi, A.; Saeki, A.; Nakaya, K.; Kato, T.; Ito, K.; Amemiya, Y. Phys. Rev. E 2000, 62, 6874.

(42) Baciu, M.; Olsson, U.; Leaver, M.; Holmes, M. J. Phys. Chem. B 2006, 110,16 . 


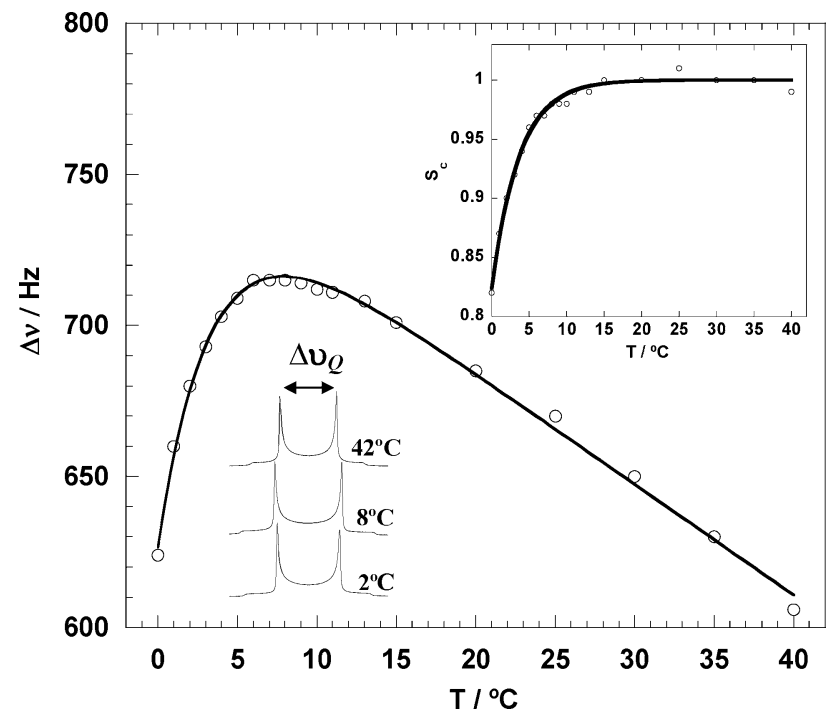

Figure 4. Quadrupolar splittings, $\Delta v_{\mathrm{Q}}$, from $\mathrm{D}_{2} \mathrm{O}$ vs temperature. The solid line is the best fit of eq 2 to the data. As inserts, we also show ${ }^{2} \mathrm{H}$ NMR spectra recorded at 2,8 , and $42{ }^{\circ} \mathrm{C}$, respectively (bottom), and in the upper right corner the variation with temperature of the extracted order parameter, $S_{\mathrm{c}}$ (see text for details).

surfactant layer. Near $10^{\circ} \mathrm{C}$, however, $\Delta v_{\mathrm{Q}}$ shows a maximum, and a further decrease of the temperature results in a strong decrease of $\Delta v_{\mathrm{Q}}$. Such a decrease has been found to correlate with the formation of a mesh structure. ${ }^{42}$ In a two-state discrete exchange model, $\Delta v_{\mathrm{Q}}$ depends on (i) the fraction of water molecules, $P_{\mathrm{b}}$, that are perturbed by the bilayer-water interfaces, (ii) the local orientational ordering in the perturbed "hydration layer", characterized, e.g., by a "reference" quadrupolar splitting, $\Delta v_{\mathrm{Q}}^{0}$, and (iii) how the interface is curved in space. Molecular diffusion along a curved surface results in an averaging of the quadrupolar interaction. The partial averaging due to diffusion over the locally curved interface in a perforation can be characterized by the order parameter, $S_{\mathrm{c}}$, bound by $0 \leq S_{\mathrm{c}} \leq 1$, where $S_{\mathrm{c}}=1$ corresponds to the absence of perforations and $S_{\mathrm{c}}$ $=0$ to a completely isotropic distribution of orientations sampled by the lateral interfacial diffusion. With these assumptions, we can write

$$
\Delta v_{\mathrm{Q}}(T)=P_{\mathrm{b}}(T) \Delta v_{\mathrm{Q}}^{0}(T) S_{\mathrm{c}}(T)
$$

where all parameters are temperature-dependent. The parameters are, however, expected to vary monotonically with temperature. $P_{\mathrm{b}}$ and $\Delta v_{\mathrm{Q}}^{0}$ are expected to decrease with increasing temperature. The non-monotonic variation of $\Delta v_{\mathrm{Q}}$ then implies that $S_{\mathrm{c}}$ increases with increasing temperature but levels off above a certain temperature, here $\sim 10{ }^{\circ} \mathrm{C}$. At higher temperatures, where $S_{\mathrm{c}} \approx$ $1, \Delta v_{\mathrm{Q}}$ decreases approximately linearly with increasing temperature. Assuming a linear dependence in the whole temperature range, we can find the variation of $S_{\mathrm{c}}$ by comparing the measured $\Delta v_{\mathrm{Q}}$ with values obtained by extrapolation from the hightemperature data. A reasonable test function for $S_{\mathrm{c}}$ is an exponential, $S_{\mathrm{c}}=1-c \exp \left\{-T / T_{\mathrm{c}}\right\}$, where $c$ is a constant and $T_{\mathrm{c}}$ is a characteristic temperature, below which perforations occur. Assuming this function for $S_{\mathrm{c}}$ and a linear temperature dependence for the product $P_{\mathrm{b}} \Delta v_{\mathrm{Q}}^{0}$, we have fitted the following function

$$
\Delta v_{\mathrm{Q}}(T)=(a-b T)\left(1-c \exp \left\{-T / T_{\mathrm{c}}\right\}\right)
$$

to the data in Figure 4. The best fit is shown as a solid line, and as can be seen, it describes the data well. This is a fit with four free parameters, and we of course should not overemphasize its

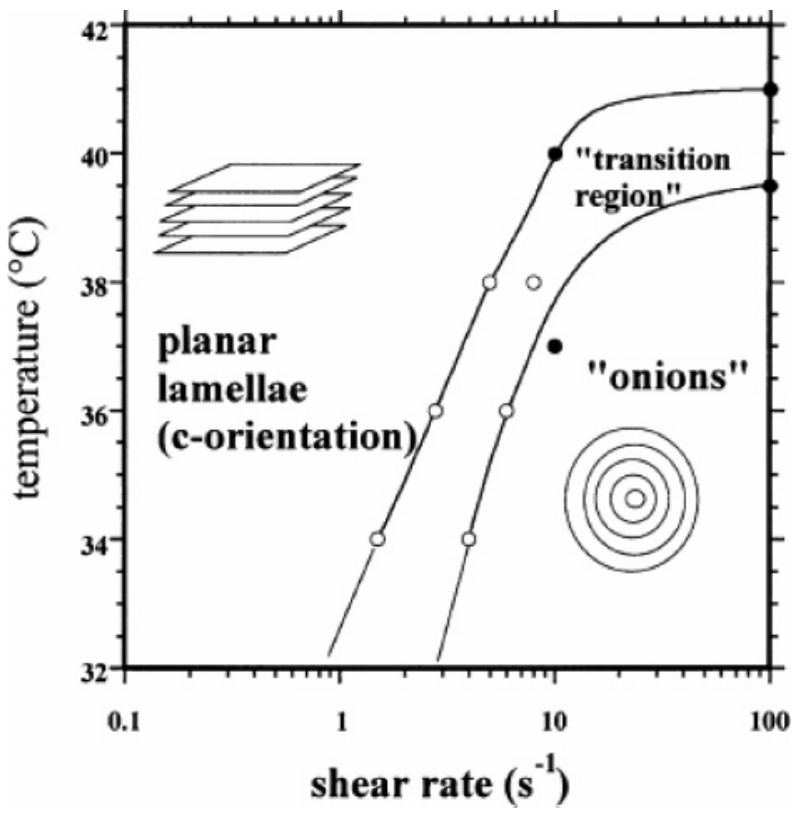

Figure 5. Partial dynamic phase diagram for the system of $40 \mathrm{wt}$ $\% \mathrm{C}_{10} \mathrm{E}_{3}$ in $\mathrm{D}_{2} \mathrm{O}$ Open symbols represent boundaries determined from viscosity data, and filled symbols were taken from rheo-SANS experiments (figure adapted from ref 28).

significance. The values of the fit parameters are as follows: $a$ $=757.4 \mathrm{~Hz}, b=3.663 \mathrm{~Hz} /{ }^{\circ} \mathrm{C}, c=0.1726$, and $T_{\mathrm{c}}=3.663{ }^{\circ} \mathrm{C}$. We stress, nevertheless, that the non-monotonic temperature variation of $\Delta v_{\mathrm{Q}}$ can be explained by two terms: one which decays weakly with temperature, which we in fact expect due to local thermal motions, and one increasing function which levels off at higher temperatures. The exponential function for $S_{\mathrm{c}}$ obtained from the fit is shown as an insert (upper right corner) in Figure 4.

On the Shear-Induced Onion Formation. Shear-induced onion formation has been studied extensively in the present system. For a $40 \mathrm{wt} \%$ sample, the lamellar phase in C-orientation is stable in shear flow for temperatures of $>40{ }^{\circ} \mathrm{C}$. At lower temperatures, the lamellar structure is unstable in shear flow, and onion formation already occurs at a few reciprocal seconds. The stability diagram has been determined previously ${ }^{28}$ and is shown in Figure 5(reprinted from Oliviero, C.; Coppola, L.; Gianferri, R.; Nicotera, I.; Olsson, U. Colloids Surf., A: Physicochem. Eng. Aspects 2003, 228, 85, with permission from Elsevier).

The major objective of the present study was to investigate whether there exists a connection between the possibility of forming onions and the presence and stability of screw dislocations and bilayer perforations. Bilayer perforations and a random mesh phase seem to occur, but only below $10^{\circ} \mathrm{C}$. Bilayer perforation therefore cannot play a role in the observed onion formation at 20-30 ${ }^{\circ} \mathrm{C}$. We have found that the lamellar phase shows essentially an elastic response to a small shear deformation in the frequency range $0.5-5 \mathrm{~Hz}$. The elastic modulus increases strongly with decreasing temperature. We attribute the elasticity to screw dislocations, connecting adjacent layers in the bilayer stack. The strong temperature dependence of the elastic modulus then suggests that the screw density increases with decreasing temperature. An increasing screw density with decreasing temperature has been observed in a similar system. ${ }^{22}$

Nonionic surfactants of the ethylene oxide kind are special in some ways. They may show a number of phase and structural transitions when varying the temperature that are not simple order-disorder transitions but rather reflect that the spontaneous 
monolayer curvature, $H_{0}$, varies strongly with temperature. ${ }^{43}$ At lower temperatures, $H_{0}>0$, while the opposite holds for higher temperatures. At some particular temperature, $T_{0}, H_{0}=0$. This occurs approximately at the temperature when a dilute lamellar phase transforms to the sponge phase, ${ }^{44}$ which in the present case is approximately $30^{\circ} \mathrm{C}$.

Lamellar phases often occupy larger areas in phase diagrams than neighboring hexagonal or cubic phases. The reason for its "success" is the following. The three- and two-dimensionally ordered cubic and hexagonal phases do not allow large structural variations before losing their symmetry. The one-dimensionally ordered lamellar phase, on the other hand, may sustain larger fluctuations (we may also compare the melting points of the different phases; for a collection of phase diagrams see, e.g., Mitchell et al. $)^{45}$ and more structural defects without losing its one-dimensional order. Thus, it is not surprising when systematic structural variations are observed within a lamellar phase, in particular, when varying the temperature in a nonionic surfactant system.

Returning to the problem of why onions often, but not always, form when shearing a lamellar phase, we may conclude that the

(43) Olsson, U.; Wennerstrom, H. Adv. Colloid Interface Sci. 1994, 49, 113. (44) Le, T. D. In Doctoral Dissertation, Physical Chemistry 1; University of Lund, 2000.

(45) Mitchell, D. J.; Tiddy, G. J. T.; Waring, L.; Bostock, T.; McDonald, M. P. J. Chem. Soc., Faraday Trans. I 1983, 79, 975. bilayer perforations and the random mesh phases do not correlate with onion stability, as already stated above. The observed elastic response to small-amplitude shear deformations and the strong temperature dependence of the elastic modulus is striking. The observation is robust, in that it is reproducible and reversible when cycling the temperature, demonstrating that layers in the stack are connected, presumably by screw dislocations. It is possible that these screw dislocations, the density of which increases as the temperature is lowered, play an important role in the flow instability of the lamellar C-orientation and the shearinduced formation of multilamellar vesicles.

Acknowledgment. U.O. thanks the Yukawa Institute for Theoretical Physics at Kyoto University and Masayuki Imai and Shigeyuki Komura for stimulating discussions during the YITP workshop "Structures and Dynamics in Soft Matter - Beyond Self-Organization and Hierarchical Structures" in July 2006. We also thank Seyda Bucak for comments on the manuscript. The Colloid Group in Coimbra University is supported by grants from the Fundação para a Ciência e Tecnologia (FCT) (projects ref: POCTI/QUI/45344/2002 and POCTI/QUI/58689/2004). Bruno Medronho acknowledges Fundação para a Ciência e Tecnologia (FCT) for a research grant (ref SFRH/BD/21467/ 2005). We acknowledge the Swedish Research Council for financial support.

LA063599A 